\title{
Initial evaluation of a practical PET respiratory motion correction method in clinical simultaneous PET/MRI
}

\author{
Richard Manber ${ }^{1 *}$, Kris Thielemans ${ }^{1}$, Brian Hutton ${ }^{1}$, Anna Barnes ${ }^{2}$, Sebastien Ourselin ${ }^{1}$, Simon Arridge ${ }^{1}$, \\ Celia O'Meara ${ }^{2}$, David Atkinson ${ }^{1}$ \\ From PSMR14: 3rd Conference in PET/MR and SPECT/MR \\ Kos Island, Greece. 19-21 May 2014
}

${ }^{1}$ University College London, London, UK
Respiratory motion during PET acquisitions can cause image artefacts, with sharpness and tracer quantification adversely affected due to count 'smearing'. Motion correction by registration of PET gates becomes increasingly difficult with shorter scan times and less counts. The advent of simultaneous PET/MRI scanners allows the use of high spatial resolution MRI to capture motion states during respiration [1,2]. In this work, we use a respiratory signal derived from the PET list-mode data [3,4], with no requirement for an external device or MR sequence modifications.

Clinical PET data are grouped into 10 respiratory bins based on respiratory signal amplitude derived from the PET list-mode data (Deep breaths outside defined limits are ignored) (Figure 1). During an extra post-scan 30s PET/MRI acquisition, rapid 2D Gradient Echo MR images are collected and grouped into these 10 respiratory bins. Images in each bin are averaged to form one image per bin (Figure 2), which are registered to a reference image, forming a patient-specific motion model. Motion estimates from the model are applied directly within the reconstruction of the clinical PET list-mode data using Motion Compensated Image Reconstruction (MCIR) [5], to form one motion-corrected image. On two human subjects $\left({ }^{18} \mathrm{~F}\right.$-FDG - 1 multiple liver lesions, 1 cardiac) we present PET data motion-corrected with an MRI motion model. Images are assessed visually, with line profiles through ROIs (Figure 3), and by change of pixel intensity in regions of high activity.

In the liver case we see a decrease in tumor 'smearing' after MRI model-based correction (Figure 3). Other areas of high activity in the liver, only marginally visible in the uncorrected image, become apparent in the motion-corrected image. Average intensity increase over the 3 lesions is $11 \%$, while increase in intensity in the cardiac wall in the cardiac patient is $10 \%$.

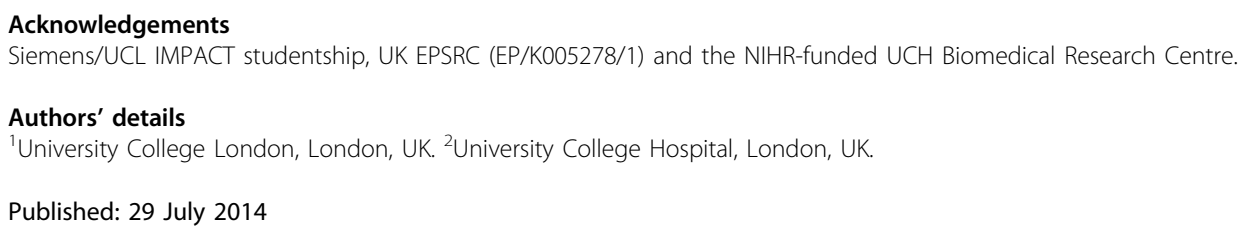

C 2014 Manber et al; licensee Springer This is an Open Access article distributed under the terms of the Creative Commons Attribution License (http://creativecommons.org/licenses/by/4.0), which permits unrestricted use, distribution, and reproduction in any medium, provided the original work is properly cited. 


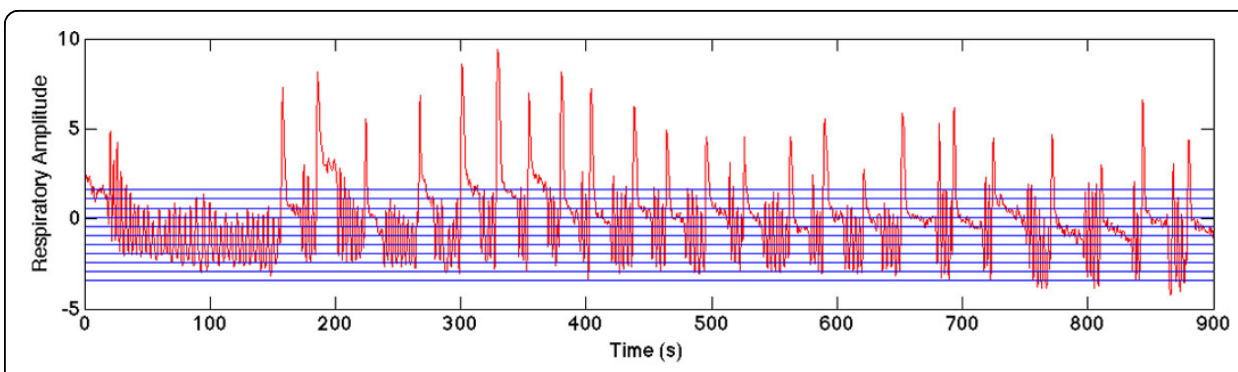

Figure 1 Respiratory signal throughout clinical 15 minute PET acquisition, including 'free breathing' and 'breath-hold' sections. Horizontal lines used to bin the PET data based on signal amplitude are also shown. Deep inhalation periods are excluded.

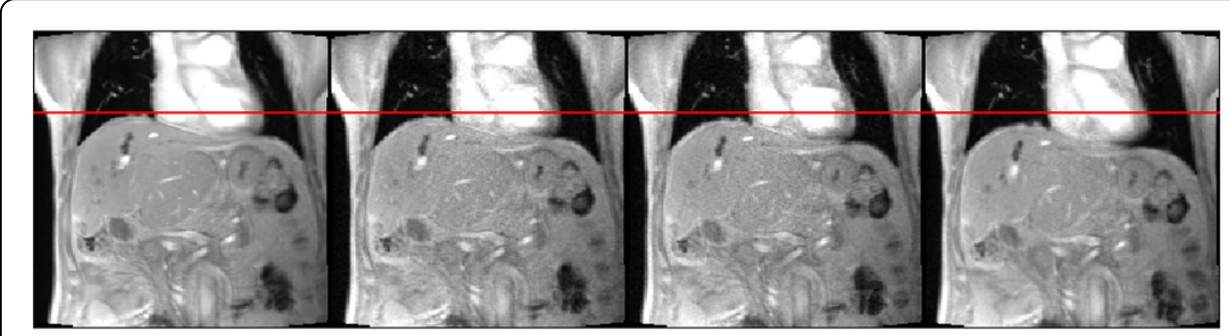

Figure 2 Binned and averaged MRI slices for 4 out of the 10 bins; ranging from end-expiration to endinspiration.
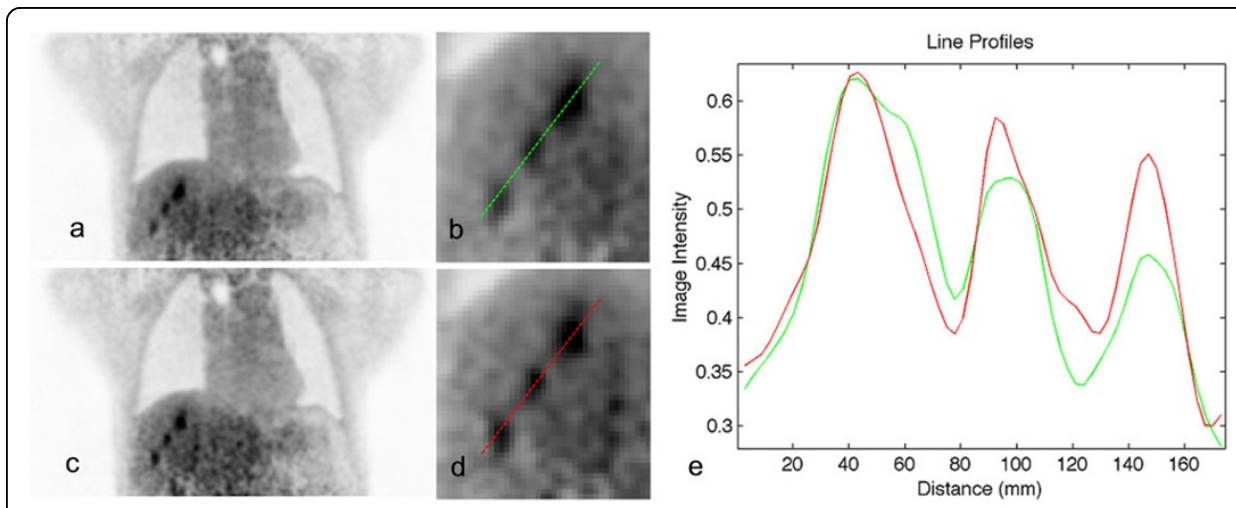

Figure 3 Uncorrected image (a) with $\mathrm{ROI}$ (b), motion-corrected image (c) with $\mathrm{ROI}$ (d), line profiles through the liver lesions in both images (e). Image intensity scale is in arbitrary units.

\section{References}

1. Chun SY, et al: MRI-based nonrigid motion correction in simultaneous PET/MRI. J NuCl Med 2012, 53(1):1284-91.

2. King AP, et al: Thoracic respiratory motion estimation from MRI using a statistical model and a 2-D image navigator. Medical Image Analysis 2012, 16:252-264.

3. Thielemans K, et al: Device-less gating for PET/CT using PCA. Nuclear Science Symposium and Medical Imaging Conference (NSS/MIC) 2011, 3904-3910.

4. Manber, et al: ISMRM Conf. Proc 2014

5. Polycarpou I, et al: Analysis and comparison of two methods for motion correction in PET imaging. Medical Physics 2012, 39:6374-6483.

6. Thielemans K, et al: STIR: software for tomographic image reconstruction release 2. Phys Med Biol 2012, 57(4):867-883.

\section{doi:10.1186/2197-7364-1-S1-A40}

Cite this article as: Manber et al:: Initial evaluation of a practical PET respiratory motion correction method in clinical simultaneous PET/MRI. EJNMMI Physics 2014 1(Suppl 1):A40. 